\title{
AC 2011-235: USING KEFIR TO TEACH MICROBIAL KINETICS IN AN UNDERGRADUATE WASTEWATER TREATMENT COURSE
}

\section{Isaac W. Wait, Marshall University}

Isaac W. Wait is an assistant professor of engineering in the College of Information Technology and Engineering at Marshall University in Huntington, West Virginia. Dr. Wait conducts research and teaches courses in the area of water resources and environmental engineering, and is a registered Professional Engineer in the States of Ohio and West Virginia.

\section{Richard F. McCormick, Marshall University}

Richard F. McCormick is a Professor of Engineering at Marshall University in Huntington, West Virginia. He received his BSCE from WV Tech in 1971 and MS and PhD from Va Tech in 1973 and 1979. After teaching for 28 years at WV Tech, he moved to Marshall in 2003 where he has helped to start a new program in engineering which was recently accredited by ABET. His research interests have been in physical and chemical treatment of water and wastewater and his teaching interests have been in enviromental and water resources engineering, engineering mechanics and surveying. He is a registered professional engineer and a professional surveyor in West Virginia. He and his wife Anna live in Saint Albans, West Virginia and have three children and three grandchildren.

Sydney M Wait, Marshall University 


\title{
Using kefir to teach microbial kinetics in an undergraduate wastewater treatment course
}

\begin{abstract}
As a method of providing hands-on experience in learning reaction kinetics, undergraduate engineering students enrolled in a wastewater treatment course were assigned to characterize the kinetics of kefir production in order to design hypothetical biological reactors. Students designed and implemented experiments investigating parameters including the effects of change in temperature, DO, F:M ratio, $\mathrm{pH}$, the presence of inhibitory substances, and other factors. Kefir is a fermented milk drink that is safe to handle and easy for students to produce, and provides instructional analogies to aspects of biological growth that can be applied when teaching students about wastewater treatment.

Student teams were supplied with kefir grains, and were responsible for the ongoing growth and survival of kefir colonies. Changes were made to growth conditions during experimentation (e.g., temperature, oxygen availability, mixing, addition of simple sugars to solution, etc.) in order to observe the effect on kefir production rate, and students measured a variety of indicators of process performance (e.g., $\mathrm{pH}$, degree of carbonation, separation of milk solids, etc.). Students fit data to kinetic models, and derived growth constants that were then used in the design of a scaled kefir production process. Feedback gathered from students indicated enthusiasm with the project and enhanced understanding of reaction kinetics. Gaining independent, self-directed experience in the lab provided students with important opportunities to practice critical thinking and experience the independent reasoning that is required for success beyond graduation.
\end{abstract}

\section{Introduction}

Reaction kinetics is often taught at the beginning of undergraduate environmental engineering courses in order to help students understand temporal relationships in processes such as drinking water disinfection, secondary treatment of wastewater, and remediation of contaminated soil. Although some students intuitively understand concepts without physical examples, others find reaction kinetics abstract and difficult to grasp in the absence of visual observation or kinesthetic manipulation of the variables that influence reaction rate. Traditionally it has been challenging to give students learning activities that enable them to observe and manipulate biological systems, particularly in the case of wastewater treatment. It is difficult in an undergraduate academic environment to work with pathogenic microorganisms derived from wastewater samples due to concerns over student infection, the inconvenience associated with providing substrate (i.e., wastewater), and difficulties associated with providing students with unrestrained lab access in 
order to take frequent measurements and make adjustments to growing conditions. Thus, it is clear that an alternative which eliminates these challenges would be an advancement towards giving students the opportunity to have hands-on experience with biological growth, and better understand the factors that influence reaction kinetics.

Kefir is a drink that originates from the Caucus region of Eurasia, and is similar in appearance and taste to unsweetened, drinkable yogurt. The production of kefir depends on the presence of kefir grains (see Figure 1), which are colonies of bacteria and yeast that live in jelly-like clusters and are placed into milk during kefir production ${ }^{1}$. The growth of kefir, wherein microorganisms consume a substrate (lactose present in milk), generate a by-product (kefir), and increase in cellular mass ${ }^{2}$, has analogues to suspended growth secondary treatment of wastewater. Yeasts present in kefir include Saccharomyces delbrueckii, Sacc. Cerevisiae, and Sacc. Exiguous, and bacteria present in kefir include Lactobacillus caucasicus and Lact. Kefir ${ }^{3,4}$. Like the reduction of biochemical oxygen demand (BOD) by bacteria in secondary treatment aeration basins, kefir production is affected by factors such as temperature, concentration of dissolved oxygen (DO), food-to-microorganism (F:M) ratio, $\mathrm{pH}$, mixing, and the presence of inhibitory substances such as inorganic and organic contaminants. However, since the bacteria and yeasts in kefir are not pathogenic, it is possible for undergraduate students to handle and manipulate samples without concerns about becoming sick, and since the substrate required for kefir growth is milk, the unpleasant handling of wastewater samples is avoided.

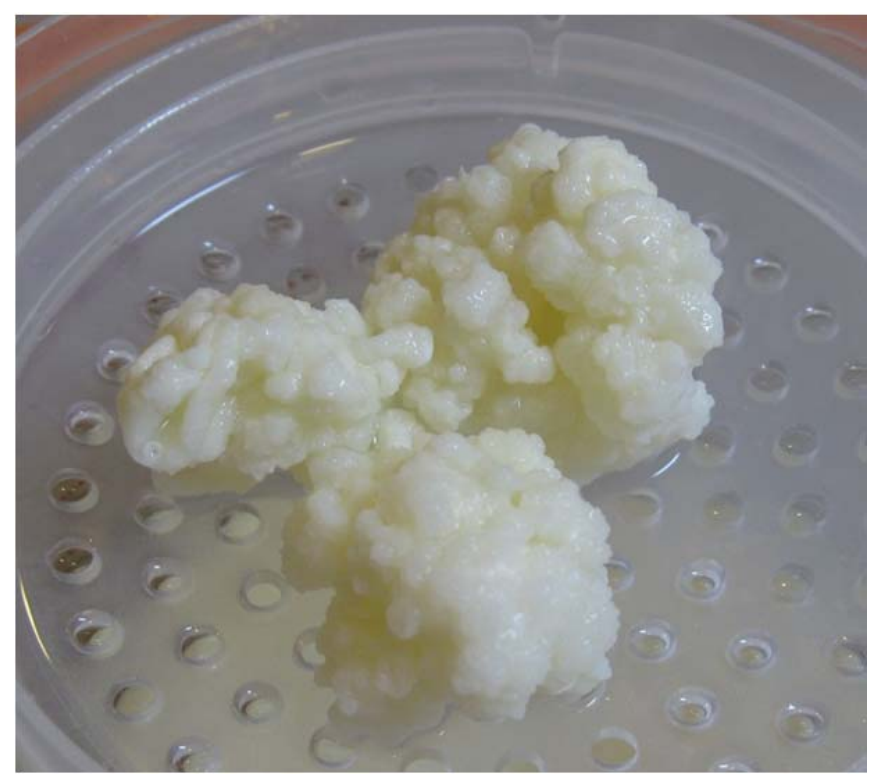

Figure 1 - Photograph of kefir grains, which are responsible for the fermentation of milk into kefir.

In addition to providing an opportunity for students to see and manipulate biological reaction kinetics, an interactive team-based project such as described herein also provides students with 
the opportunity to design and document experiments, develop new experimental methods, manage responsibilities and communications in a team environment, and experience the demands associated with primary responsibility of an ongoing, multi-day experiment. This last objective is particularly useful for students who are considering graduate school, since many undergraduate students have not yet had experience in taking on responsibility for the success of experiments beyond the confines of a scheduled lab period. Problem-based learning activities in the context of a wastewater treatment course have been identified as a particularly effective way of achieving program outcomes ${ }^{5}$, with a primary advantage of such activities being increased student engagement and higher level problem solving and communications.

\section{Project Approach}

The pedagogical approach that was undertaken when conducting a "Microbial Kinetics and Reactor Design” project during the Spring 2010 offering of Advanced Water and Wastewater Treatment (CE 434) at Marshall University is summarized in the sections below. CE 434 is a senior-level, elective undergraduate course without a lab component, and it is taken by students as a follow-on to an earlier required undergraduate water and wastewater treatment course that does include a laboratory component. During the Spring 2010 semester, eight students were enrolled in CE 434, and they were assigned into two groups by the instructor for purposes of conducting this project.

\section{Project problem statement}

Midway through the semester, after having already covered concepts related to kinetics, reactor theory, and secondary treatment of wastewater, the following problem statement was given to student groups:

Your team has been hired by a local dairy to provide a report on how to begin commercial kefir production. You will be provided with active kefir grains, and with those grains you must characterize the growth kinetics of kefir under a variety of environmental conditions. Once kinetics have been characterized, and an optimal production "recipe" has been established, you must design a large-scale process to generate 50,000 servings of kefir per day. Consider batch reactor processes, CMFR processes, and PFR processes.

Essentially the project has two phases: (1) to characterize the growth rates of kefir under a variety of different conditions (while simultaneously assessing the 'quality' of the finished kefir under these conditions), and (2) to apply kinetics relationships in reactor models in order to design a larger-scale process. The primary analogy being utilized in this project is that the 
microbes that produce kefir grow in many of the same ways that the microbes that break down BOD in wastewater treatment. Although the second part of this project was to design a kefir production process once kinetics had been established, the primary analogy could instead be applied to a scenario wherein students are directed to size a wastewater treatment facility once microbial kinetics have been characterized.

\section{Project objectives and concepts taught}

In order to guide student expectations and help explain why the project was being carried out, the following project objectives were provided to students:

1. Increase familiarity with engineering and scientific literature search.

2. Gain experience designing experiments.

3. Improve understanding of factors that influence microbial growth kinetics.

4. Apply kinetics to reactor design.

5. Employ teamwork, communication skills, and time management.

Enhanced understanding of numerical models, and increased ability to independently design and conduct experiments are among the pedagogical benefits to conducting a project that requires students to design their own bench-scale experiments utilizing microbial cultures.

\section{Numerical models}

The concept of numerical models can often be vague and abstract to some students, and while graphical representation of a system's performance can help to make these abstract ideas more concrete for some students, there is no substitute for having students directly observe, manipulate, and personally measure a physical system, and then construct their own numerical model to describe the performance of that system in response to changes in input parameters. Since numerical models are increasingly relied upon in all areas of engineering design and analysis, it is critical that students have experiences that help them learn how models are assembled, how they can be useful, and what their limitations may be.

\section{Independent experiment design}

Although undergraduate engineering students typically take several courses with a laboratory component, most of the labs that students undertake are of the variety where a pre-defined procedure is provided to the student, equipment resources are identified in advance and instructions are issued on how to use them, and minimal original or critical thinking is required for students to generate the data that is required. In many cases, students are specifically told how to interrogate the data that is collected (e.g., which plots to generate, what trends to look for, etc.). While such specificity may be important for purposes of efficiency where many labs during a semester must be conducted and may help students to gain basic familiarity with how to 
conduct lab experiments, it does not provide students with strong opportunities to personally design procedure. Furthermore they lack the understanding that comes from observing responses to changes in independent variables and their effect on experimental outcomes within the designed procedure.

A more suitable environment is provided for students to utilize and develop critical thinking skills when they are instructed in the fundamental concepts that influence a system's behavior, guided in determining what independent variables should be investigated through experimentation, and pointed toward the laboratory equipment they will need to conduct the experiments. In this project, students were responsible for: (a) identifying the input parameters that would be investigated, (b) identifying existing analytical methods for characterizing dependent variables, (c) developing new analytical methods for characterizing behavior where an existing formalized approach did not exist, (d) creating an experimental matrix that summarized the key parameters under evaluation during each run of the experiment, and (e) developing suitable datasheets, lab notebooks, and other data storage methods for collecting and preserving the results of experimentation. Because students are typically unfamiliar with some or all of these more independent activities, in-class discussions were held to stimulate student thinking about what was needed in each category. Requiring periodic draft submissions provided opportunity for feedback in a low-stakes environment.

\section{Investigation parameters}

After providing students with background information about kefir, and explaining the analogy between the growth of kefir microbes and the growth of microbes in a wastewater secondary treatment environment, a classroom discussion was held to elicit student input about which factors should be characterized relative to the impact on kinetics. Among the input factors identified were:

- Temperature

- Concentration of dissolved oxygen

- Effect of sequencing dissolved oxygen (periods of anoxic conditions followed by aerobic conditions)

- $\quad$ Starting $\mathrm{pH}$ of the mixture

- Ratio of food-to-microorganisms (F:M ratio)

- Mixing

- Milkfat content

- Presence of inhibitory substances (e.g., metals)

After determining independent variables, students identified the following parameters (dependent variables) as possible indicators of process performance: 
- Dissolved oxygen concentration

- $\mathrm{pH}$ of the mixture over time

- Viscosity of the end-product

- Degree of whey / water separation

- Amount of carbonation

- Specific gravity of the end-product (reflective of alcohol content)

- Flavor

It is with the student-discussion-derived list of independent and dependent variables that student groups were tasked to develop experimental matrices and identify / develop the needed experimental methods.

\section{Resources and additional guidance given}

Since one of the objectives of this project was to enhance student familiarity with the process of and need for scientific literature search, an in-class demonstration was conducted to familiarize students with the article databases available through the university library. Students were expected to include citations to peer-reviewed journal articles in their final reports, the objective of this being that students would get a better sense for both the scientific experimental process and the typical means of communicating experimental results in the literature through reviewing these publications.

The laboratory equipment requirements for conducting such a project are relatively modest, and in fact could be tailored to permit students to conduct the experiment in their own homes, which could be important depending on the frequency of data sampling and how often the kefir production process cycles. The principal requirements are: kefir grains, a non-metallic container, milk, volumetric flask, non-metallic strainer, and a $\mathrm{pH}$ meter. Additional equipment items that may improve experiment results and enhance the variety of data that can be collected include: a constant-temperature environment (e.g., incubator, constant temperature water bath, etc.), magnetic stir bars and stir plate, centrifuge, dissolved oxygen meter, and electronic balance for determining the mass of kefir grains. 


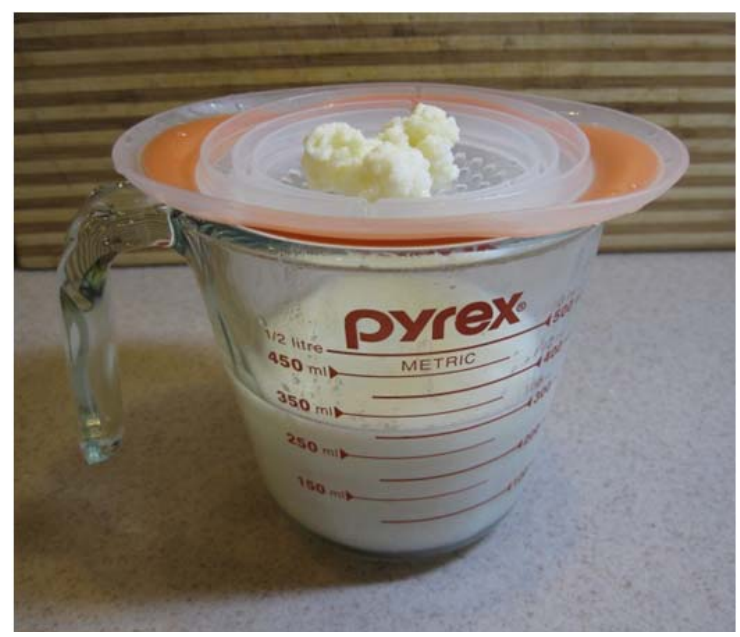

Figure 2 - Photograph illustrating the method used for separating finished kefir from kefir grains at the completion of experiments.

Kefir should be produced in a constant temperature environment free from contamination by external particles. Degree of mixing, how well-sealed the production chamber is during fermentation, and the ratio of milk-to-kefir (F:M) are all factors suitable for student examination even in locations outside of the standard educational lab environment (i.e., in students' homes). Where daily lab access is available to students, additional complexity can be added to experimentation to allow additional factors to be interrogated.

One of the end-product parameters of the kefir fermentation reaction that students can measure as an indicator of reaction rate is the quantity of alcohol that has been produced. The alcohol content of kefir increases with the degree to which it is isolated from oxygen during fermentation, and the length of time that the fermentation is allowed to continue. The typical range of alcohol content in finished kefir ranges from $0.06 \%$ to a maximum of $3 \%$ when anaerobic conditions are carefully controlled (Marshall and Cole, 1985). Measuring alcohol content is most direct and accurate when a gas chromatograph (GC) is available, although measuring changes in density of the solution with a specific gravity hydrometer (such as VWR Catalog 35123-426) can also be used.

\section{Student results}

Of the experimental conditions that change during milk fermentation, perhaps the easiest for students to monitor over time is $\mathrm{pH}$. Milk taken from a commercial container has a starting $\mathrm{pH}$ of approximately 7.0, and the milk/kefir grain mixture becomes more acidic over time as fermentation proceeds. As shown in Figure 3, the decrease in $\mathrm{pH}$ of milk during kefir production follows a curve consistent with behavior observed during first-order kinetics. 
During the milk fermentation process, milk solids separate from the water component of milk. Through centrifugation of the final product of the kefir production process, it is possible to quantify the amount of milk solids that have separated out from the original milk solution. This method is conceptually analogous to determining the settling properties of activated sludge by determining Sludge Volume Index (SVI). Through settling of milk solids over a specified duration and at a specific rotational speed, it can easily be determined how far into the fermentation process a certain sample has proceeded.

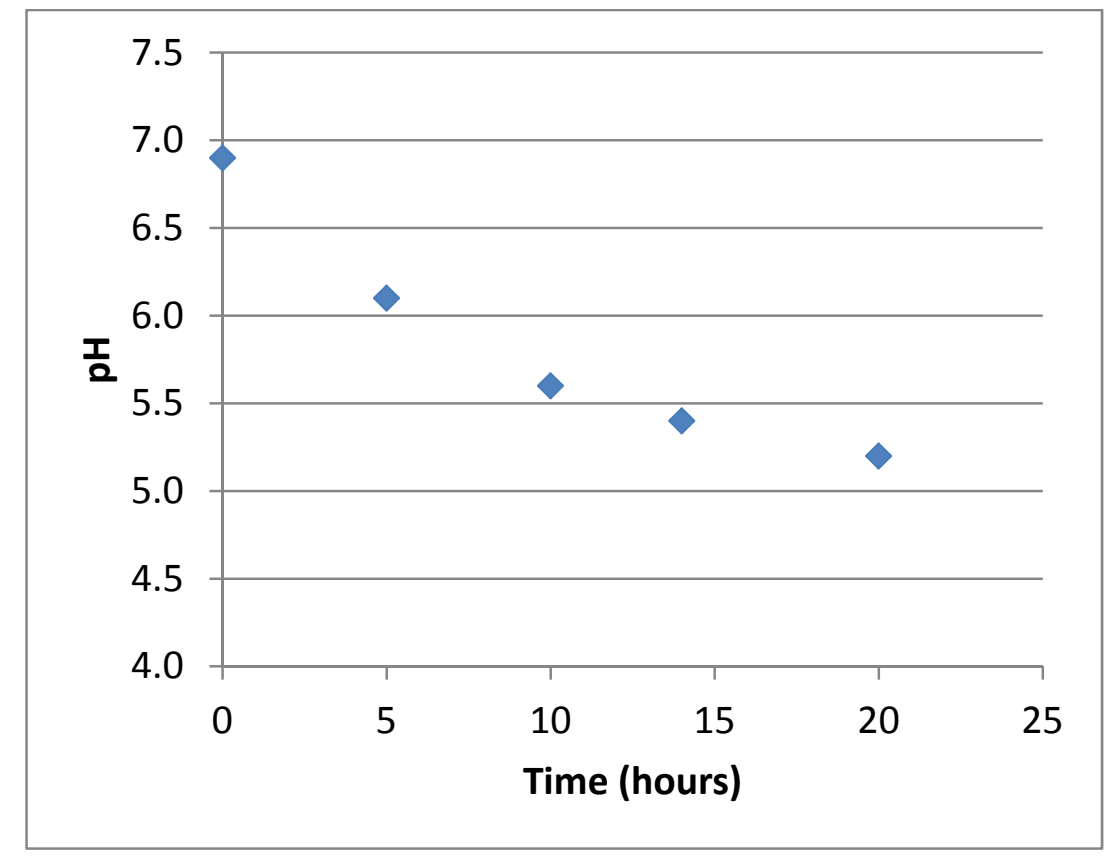

Figure 3 - Student results showing decrease in solution $\mathrm{pH}$ during kefir production.

A method for using a centrifuge to determine the degree to which the kefir production reactions had proceeded was developed by one of the student groups in this project. Although a centrifuge was present in the lab area where students conducted their experiments, its use was not suggested by the instructor. Beyond executing a given laboratory procedure, and beyond determining which lab procedure is suitable in given circumstances, the independent development of an altogether new approach for quantifying the temporal distribution of dependent variables is evidence of critical and independent thought in students, and was for this activity one of the most direct evidences of achievement of the objectives related to independent problem solving.

Likewise, students developed their own approaches to such problems as how to control the amount of dissolved oxygen present in the milk during the fermentation process. One student group purchased and modified a fish aquarium airstone in order to provide an excess of oxygen to milk during their 'aerobic' experimental runs. Over the course of experimentation, this 
airstone system was modified to work in a constant-temperature incubator, and in conjunction with student-developed solutions to ensure mixing of the substrate. Students achieved anoxic conditions by (independently) familiarizing themselves with an available vacuum pump, and applying vacuum conditions to the headspace above a container of milk prior to inoculation with the kefir grains. When observing that milk left standing would also experience a pH change even when not inoculated with kefir grains, students identified the need for a control sample wherein $\mathrm{pH}$ changes attributable to fermentation could be separated from $\mathrm{pH}$ changes due to interaction with atmospheric gases (i.e., carbon dioxide). Without any direct involvement of the instructor, students familiarized themselves with how to operate (and calibrate) a new $\mathrm{pH}$ meter that they had not before operated, a spectrophotometer, and hydrometers. In general, students showed a remarkable degree of independence, initiative, intellectual curiosity, and creativity in the ways that they manipulated biological samples in order to observe effects on growth rate and other output parameters.

\section{Conclusions}

By providing students with kefir grains and simple lab resources typically available in an environmental engineering laboratory, students were able to experiment with kefir to investigate the following:

- Relationship between increasing temperature and increasing rate of kefir production

- Impact on rate of reaction of providing sugars to the substrate solution

- Variation on reaction rate according to adjustment of F:M ratio

- Conditions required to increase the degree of carbonization of end-product

Achievement of the activity's learning objectives was demonstrated by student identification of factors that could affect microbial kinetics, formulation of experimental matrices, development of new experimental methods, and execution of a multi-week experimental plan that required organization, teamwork, and careful attention to keep biological samples viable.

\section{Student comments in course evaluations}

At the conclusion of the project, students were asked to complete a ten-question survey to assess the impact of the project. The results of multiple-choice questions from the survey are shown in Tables 1 and 2. 
Table 1 - Results from multiple-choice questions to assess completion of learning objectives, included in survey of students following the project.

\begin{tabular}{|l|c|c|c|c|c|}
\hline \multicolumn{1}{|c|}{ Question } & SA & A & N & D & SD \\
\hline $\begin{array}{l}\text { This project helped me to have an "increased } \\
\text { familiarity with engineering and scientific literature } \\
\text { search." }\end{array}$ & 1 & 6 & 0 & 0 & 0 \\
\hline $\begin{array}{l}\text { This project helped me to "gain experience } \\
\text { designing experiments." }\end{array}$ & 4 & 3 & 0 & 0 & 0 \\
\hline $\begin{array}{l}\text { This project helped me to have an "improved } \\
\text { understanding of factors that influence microbial } \\
\text { growth kinetics." }\end{array}$ & 2 & 4 & 1 & 0 & 0 \\
\hline $\begin{array}{l}\text { This project helped me to "apply principles of } \\
\text { kinetics to reactor design." }\end{array}$ & 2 & 2 & 3 & 0 & 0 \\
\hline $\begin{array}{l}\text { This project was an effective opportunity to practice } \\
\text { "teamwork, communication skills, and time } \\
\text { management." }\end{array}$ & 2 & 5 & 0 & 0 & 0 \\
\hline
\end{tabular}

Note: "SA" = Strongly Agree, “A” = Agree, "N" = Neutral, "D” = Disagree, "SD” = Strongly Disagree

Table 2 - Results from multiple-choice questions to assess student satisfaction, included in survey of students following the project.

\begin{tabular}{|l|c|c|c|c|c|}
\hline \multicolumn{1}{|c|}{ Question } & Excellent & Good & Fair & Poor & Horrible \\
\hline $\begin{array}{l}\text { Overall, how would you rate this } \\
\text { project, in terms of how much you } \\
\text { learned? }\end{array}$ & 2 & 5 & 0 & 0 & 0 \\
\hline $\begin{array}{l}\text { Overall, how would you rate this } \\
\text { project, in terms of how much you } \\
\text { enjoyed it? }\end{array}$ & 2 & 5 & 0 & 0 & 0 \\
\hline
\end{tabular}

When asked, “What did you like about this project?” the student responses were:

- It was very interesting learning how different factors affect the growth and kinetics of the kefir grains.

- The faces of everyone who tried the kefir.

- The design process and experience

- Using the lab equipment. Designing experiments.

- The novelty 
- I liked its off the wall nature and thinking outside of the box. It's an interesting subject and I like the fact we had something to visibly see/taste at the end of the project. It beats the imaginary town pipe networks in the sense of having a real end result to the project.

- Experimenting and the lab process.

When asked, “What did you dislike about this project?” the student responses were:

- Drinking the kefir.

- Drinking the kefir.

- The machine is not sensitive enough

- The taste of kefir. Lack of assistance from other team members.

- The daily requirement of being in the lab

- I didn't like the taste, but I guess that was mitigated by its uniqueness. None the less a more enjoyable testing/tasting project may be more fun. Also the lack of reliable equipment for measurements made it difficult to be pleased with results.

- The Taste

Although student feedback indicates a negative response to tasting the kefir, it should be understood that the 'horrible taste' of the end product was a running joke through the semester, and some of the student feedback offered is likely a reference to this ongoing reference. This aside, it is felt that encouraging students to taste the end product following their experimentation is an effective means of getting them to personally connect with and observe the effects of changes to the independent variables in this experiment - it is one thing to see a chart of declining $\mathrm{pH}$ over time, but far more memorable for students to actually taste the kinetics.

When asked, "How should this project be changed in future semesters to make it a more effective learning experience for students?" the student responses were:

- Give out the project at the beginning of the semester to allow for more time to think over experiments. I thought of some more experiments after it was too late to include them in the report.

- The dissolved oxygen meter needs fixed. I think more time would allow the students to run more experiments and learn more about kefir. I also think integrating more reactor/process design related steps would make this project more realistic.

- Start it sooner in the semester so we are not rushing to finish it along with other projects.

- Provide some guidance in the lab on equipment available and using it.

- I like the project outcomes a lot. I just would have loved to have a temperature reader that was digital and averaged over time and better $\mathrm{pH}$ and $\mathrm{DO}$ meters. These things would make our measurements much more reliable. I loved the time we spent in lab doing engineering stuff. We need more of that in our program!

- Start them earlier so that more experiments can be done. 
In future semesters where this project is implemented, the course will be reorganized such that the background lectures on kinetics, reactor theory, and secondary treatment that should precede this project are earlier in the semester. This would allow the project to be implemented sooner in the semester to give students additional time. Although one student comment suggested that additional guidance be given regarding experimentation, there are tangible benefits to encouraging students to broaden their knowledge base through self-discovery, develop their own solutions, and independently learn how to use lab equipment.

\section{Bibliography}

1. Chen, T.H., Wang, S.Y., Chen, K.N., Liu, J.R., Chen, M.J. (2009). Microbiological and chemical properties of kefir manufactured by entrapped microorganisms isolated from kefir grains. Journal of Dairy Science, 92(7), 3002-3013.

2. Kwak, H.S., Park, S.K., Kim, D.S. (1996). Biostabilization of Kefir with a Nonlactose-Fermenting Yeast. Journal of Dairy Science, 79(6), 937-942.

3. Marshall, V.M, and Cole, W.M. (1985). Methods for making kefir and fermented milks based on kefir. Journal of Dairy Research, 52, 451-456.

4. Marshall, V.M., Cole, W.M., and Brooker, B.E. (1984). Observations on the structure of kefir grains and the distribution of the microflora. Journal of Applied Bacteriology, 57, 491-497.

5. $\quad$ Oakes, W., Sharvelle, S., Banks, M.K., Brock, B., Brophy, S. (2007). Achieving graduate competencies through an authentic design experience in a wastewater treatment course. AC2007-614, ASEE Annual Conference and Exposition, Honolulu, Hawaii, June 24 - 27, 2007. 